\title{
On Dwell Time Minimization for Switched Delay Systems: Free-Weighting Matrices Method
}

\author{
Ahmet Taha Koru, Akın Delibaşı and Hitay Özbay
}

\begin{abstract}
In this paper, we present a quasi-convex minimization method to calculate an upper bound of dwell-time for stability of switched delay systems. Piecewise LyapunovKrasovskii functionals are introduced and the upper bound for the derivative of Lyapunov functionals are estimated by free weighting matrices method to investigate non-switching stability of each candidate subsystems. Then, a sufficient condition for dwell-time is derived to guarantee the asymptotic stability of the switched delay system. Once these conditions are represented by a set of linear matrix inequalities (LMIs), dwell time optimization problem can be formulated as a standard quasiconvex optimization problem. Numerical examples are given to illustrate improvements over previously obtained dwell-time bounds.
\end{abstract}

Index Terms - Time delay systems, dwell time optimization, switched systems, free weighting matrices method

\section{INTRODUCTION}

A switched system is a dynamical system that includes a set of subsystems and a discrete switching event between those subsystems. General behaviour of a switched system is governed by following differential equation:

$$
\dot{x}(t)=f_{\sigma(t)}(x(t)), \quad \forall t>t_{0},
$$

where $\sigma$ denotes the switching signal which belongs to an index set. See the survey [12] for a review of the recent results and further references.

The stability analysis encountered in switched systems can be classified into three categories [13]. The first one is to find conditions that the switched systems are stable under any arbitrary switching signal [16], [4], [9]. The second one is to construct a switching signal that makes the switched system asymptotically stable [11]. The third category is the slow switching strategies such as dwell time stability or average dwell time stability for which the system is asymptotically stable [14], [8], [21]. The class of switching signals can be restricted to signals with the property that the interval between any consecutive switching times is not less than a value called the dwell time. The switched delay system is asymptotically stable if all of the candidate subsystems are asymptotically stable and the dwell time is large enough [15].

The literature is abounded with various of approaches for the stability analysis of time-delay systems, one can refer to [5] for a review on the topic. Main methods to deal

A. T. Koru is with Department of Mechatronics Engineering, Yıldız Technical University, İstanbul, Turkey akoru@yildiz.edu.tr

A. Delibaş1 is with Department of Control and Automation Engineering, Yildı Technical University, İstanbul, Turkey adelibas@yildiz.edu.tr

H. Özbay is with Department of Electrical and Electronics Engineering, Bilkent University, Ankara, Turkey hitay@bilkent.edu.tr with delay-dependent stability problems are model transformations. Stability analysis with model transformations leads to a sort of conservatism since analysis operates on the transformed system instead of the original system [5]. A less conservative approach to stability analysis is the free-weighting matrices method which does not include any model transformation of the original system [13], [6], [19].

There are recent results on dwell time stability of the switched delay systems. In [18] and [10], stability conditions, for a given average dwell time, are presented. There are some optimization based methods to calculate minimum dwell time [3], [20]. In [3], the calculation of dwell time is formulated as a semi-definite programming (SDP) in terms of LMIs. Piecewise Lyapunov-Krasovskii functionals is derived by model transformation methods. The upper bound of the derivative of the Lyapunov function is minimized which ends up with a sub-optimal solution to the dwell time minimization problem. The present paper proposes a quasiconvex optimization approach to directly minimize the dwell time for which the switched delay system is asymptotically stable. To reduce conservatism due to model transformation, we derive the stability conditions by using free weighting matrices.

The notation to be used in the paper is standard: $\mathbb{R}\left(\mathbb{R}^{+}\right.$, $\mathbb{R}_{0}^{+}$) stands for the set of real numbers (positive real numbers, non-negative real numbers), $\mathcal{C}$ is used to denote the set of differentiable continuous functions, $\mathbb{Z}^{+}$symbolizes the set of positive integers. The identity matrices are denoted by $I$. We use $X \succ 0(\succeq, \prec, \preceq 0)$ to denote a positive definite (positive-semidefinite, negative definite, negativesemidefinite) matrix. $\sigma_{\max }[X]$ and $\sigma_{\min }[X]$ denote the maximum and minimum singular values of $X$, respectively. The asterisk symbol $(*)$ denotes complex conjugate transpose of a matrix. The operator $\operatorname{diag}\left[X_{1}, X_{2}, \ldots, X_{n}\right]$ denotes a block diagonal matrix whose elements on the main block diagonal are $X_{1}, X_{2}, \ldots, X_{n}$. The norm $\|\cdot\|$ is defined as the Eucledian norm for a vector in $\mathbb{R}^{n}$ and the norm on $\mathcal{C}$ is defined as follows:

$$
|f|_{[a, b]}=\max \left\{\sup _{t \in[a, b]}\|f(t)\|, \sup _{t \in[a, b]}\|\dot{f}(t)\|\right\}
$$

\section{Preliminaries And Problem Definition}

Consider a class of switched delay system given by

$$
\begin{aligned}
& \dot{x}(t)=A_{\sigma(t)} x(t)+\bar{A}_{\sigma(t)} x\left(t-r_{\sigma(t)}(t)\right), \quad t \geq 0 \\
& x(\theta)=\varphi(\theta), \quad \forall \theta \in\left[-\tau_{\max }, 0\right]
\end{aligned}
$$


where $x(t) \in \mathbb{R}^{n}$ is the pseudo-state and $\sigma(t)$ is the piecewise switching signal such that $\sigma(t): \mathbb{R}^{+} \rightarrow \mathcal{P}$, $\mathcal{P}:=\{1,2, \ldots, m\}$ is an index set, $m \in \mathbb{Z}^{+}$is the number of subsystems and initial condition belongs to Banach space of continuous functions such that $\varphi(\cdot) \in \mathcal{C}$. Time delay, $r_{\sigma(t)}(t)$, is a time-varying differentiable function that satisfies

$$
\begin{aligned}
& 0 \leq r_{\sigma(t)}(t) \leq \tau_{\sigma(t)} \\
& \left|\dot{r}_{\sigma(t)}(t)\right| \leq d_{\sigma(t)}<1
\end{aligned}
$$

where $\tau_{\sigma(t)}, d_{\sigma(t)}>0$ are piecewise constants. We introduce the quartet

$$
\Sigma_{i}:=\left(A_{i}, \bar{A}_{i}, \tau_{i}, d_{i}\right) \in \mathbb{R}^{n \times n} \times \mathbb{R}^{n \times n} \times \mathbb{R} \times \mathbb{R}
$$

to describe the $i^{\text {th }}$ candidate subsystem of (1) and $\tau_{\max }=$ $\max _{i \in \mathcal{P}} \tau_{i}$.

Similar to [20], we modify the stability definition in [7] to switched delay system as in definition 1 .

Definition 1. We say that switched delay system is stable if there exists a function $\beta$ of class $\mathcal{K}$ such that

$$
\|x(t)\| \leq \beta\left(|x|_{\left[t_{0}-\tau_{\max }, t_{0}\right]}\right)
$$

along every solution to (1). Furthermore, switched delay system is asymptotically stable when it is stable and $\lim _{t \rightarrow \infty} x(t)=$ 0 .

Lemma 1. ([5]) Consider the non-switched linear subsystem $\Sigma_{i}$ of the system (1) for an $i \in \mathcal{P}$. Suppose $u_{i}, v_{i}$, $w_{i}: \mathbb{R}_{0}^{+} \rightarrow \mathbb{R}_{0}^{+}$are continuous, non-decreasing functions satisfying $u_{i}(0)=v_{i}(0)=0, w_{i}(s)>0$ for $s>0$. If there exists a continuous functional $V$, such that

$$
\begin{array}{rlrl}
u_{i}(\|x(t)\|) \leq & V_{i}\left(t, x_{t}\right) \leq v_{i}\left(|x|_{\left[t-\tau_{i}, t\right]}\right), & & \forall t \geq t_{0} \\
\dot{V}_{i}\left(t, x_{t}\right) \leq-w_{i}(\|x(t)\|), & \forall t \geq t_{0}
\end{array}
$$

then the solution $x=0$ of the subsystem $\Sigma_{i}$ is uniformly asymptotically stable.

Let us construct the following piecewise Lyapunov function:

$$
\begin{aligned}
V_{i}\left(t, x_{t}\right) & :=x^{T}(t) P_{i} x(t)+\int_{t-\tau_{i}}^{t} x^{T}(s) Q_{i} x(s) d s \\
& +\int_{-\tau_{i}}^{0} \int_{t+\theta}^{t} \dot{x}^{T}(s) Z_{i} \dot{x}(s) d s d \theta, \quad \forall i \in \mathcal{P}
\end{aligned}
$$

Lemma 2. Consider non-switched subsystems $\Sigma_{i}$ for $i \in$ $\mathcal{P}$ of switched system (1) with varying delays, $r_{i}(t)$. Given scalar $\tau_{i}>0$ and $d_{i}>0$ for which (2) and (3) hold, the $i^{\text {th }}$ subsystem is asymptotically stable if there exist symmetric matrices $P_{i} \succ 0, Q_{i} \succeq 0, Z_{i} \succ 0$, and

$$
\left[\begin{array}{cc}
X_{11 i} & X_{12 i} \\
* & X_{21 i}
\end{array}\right] \succeq 0
$$

and any appropriately dimensioned matrices $N_{1 i}$ and $N_{2 i}$ such that the following LMIs hold:

$$
\phi_{i}=\left[\begin{array}{ccc}
\phi_{11 i} & \phi_{12 i} & \tau_{i} A_{i}^{T} Z_{i} \\
* & \phi_{22 i} & \tau_{i} \bar{A}_{i}^{T} Z_{i} \\
* & * & -\tau_{i} Z_{i}
\end{array}\right] \prec 0
$$

$$
\psi_{i}=\left[\begin{array}{ccc}
X_{11 i} & X_{12 i} & N_{1 i} \\
* & X_{22 i} & N_{2 i} \\
* & * & Z_{i}
\end{array}\right] \succeq 0
$$

where

$$
\begin{aligned}
\phi_{11 i} & =P_{i} A_{i}+A_{i}^{T} P_{i}+N_{1 i}+N_{1 i}^{T}+Q_{i}+\tau_{i} X_{11 i}, \\
\phi_{12 i} & =P_{i} \bar{A}_{i}-N_{1 i}+N_{2 i}^{T}+\tau_{i} X_{12 i}, \\
\phi_{22 i} & =-N_{2 i}-N_{2 i}^{T}-\left(1-d_{i}\right) Q_{i}+\tau_{i} X_{22 i} .
\end{aligned}
$$

Proof. (For complete proof, see [19], page 45). The derivative of the Lyapunov function in (5) can be bounded as follows,

$$
\dot{V}_{i}\left(t, x_{t}\right) \leq \xi_{1}^{T}(t) \Xi_{i} \xi_{1}(t)-\int_{t-\tau_{i}}^{t} \xi_{2}^{T}(t, s) \psi_{i} \xi_{2}(t, s) d s
$$

where

$$
\begin{aligned}
\xi_{1}(t) & =\left[x^{T}(t), x^{T}\left(t-\tau_{i}\right)\right]^{T}, \\
\xi_{2}(t, s) & =\left[x^{T}(t), x^{T}\left(t-\tau_{i}\right), \dot{x}^{T}(s)\right]^{T}, \\
\Xi_{i} & =\left[\begin{array}{cc}
\phi_{11 i}+\tau_{i} A_{i}^{T} Z_{i} A_{i} & \phi_{12 i}+\tau_{i} A_{i}^{T} Z_{i} \bar{A}_{i} \\
* & \phi_{22 i}+\tau_{i} \bar{A}_{i}^{T} Z_{i} \bar{A}_{i}
\end{array}\right] .
\end{aligned}
$$

The variable $\phi_{i}$ in (6) is the Schur Complement of $\Xi_{i}$. Hence, $i^{\text {th }}$ subsystem is stable if both (6) and (7) hold.

Now, some specific lower and upper bounds for the Lyapunov function (5) can be given as

$$
\begin{aligned}
& u_{i}(s):=\sigma_{\min }\left[P_{i}\right] s^{2} \\
& v_{i}(s):=\left(\sigma_{\max }\left[P_{i}\right]+\tau_{i} \sigma_{\max }\left[Q_{i}\right]+\frac{1}{2} \tau_{i}^{2} \sigma_{\max }\left[Z_{i}\right]\right) s^{2}
\end{aligned}
$$

Another lower bound of the Lyapunov function with respect to norm of $\dot{x}(t)$ can be defined as

$$
u_{d_{i}}\|\dot{x}(t)\|^{2}:=\frac{1}{2} \tau_{i}^{2} \sigma_{\min }\left[Z_{i}\right]\|\dot{x}(t)\|^{2} \leq V_{i}\left(t, x_{t}\right) .
$$

\section{MAIN RESUlts}

The following proposition is a modified version of a result obtained in [3].

Proposition 1. For any non-switching subsystem $\Sigma_{i}$ satisfying lemma 1 with $\lim _{s \rightarrow \infty} u_{i}(s) \rightarrow \infty$, assume there exists a function $u_{d_{i}}$ such that

$$
u_{d_{i}}(\|\dot{x}(t)\|) \leq V_{i}\left(t, x_{t}\right)
$$

For an arbitrary $\eta, 0<\eta<\delta_{2},|x|_{\left[t_{0}-\tau_{i}, t_{0}\right]} \leq \delta_{1}<\delta_{2}$ implies

$$
|x|_{\left[t-\tau_{\max }, t\right]} \leq \eta, \quad \forall t>t_{0}+\tau_{\max }+T_{i}(\eta)
$$

where $T_{i}(\eta)=\left[v_{i}\left(\delta_{1}\right)\right] / \gamma_{i}(\eta), v_{i}$ defined as in the lemma 1 and $\gamma_{i}(\eta)=\inf _{\eta \leq s \leq \delta_{2}} w_{i}(s)$.

Proof. Let $T_{*}>0$ and let $\left\|x\left(t_{1}\right)\right\|>\eta$ for a time instant $t_{1}>t_{0}+T_{*}$. Let $\gamma_{i}(\eta)=\inf _{\eta \leq s \leq \delta_{2}} w_{i}(s)$. Since the subsystem $\Sigma_{i}$ is stable and $V_{i}$ is a Lyapunov-Krasovskii functional, from lemma 1 , we have the following

$$
\dot{V}_{i}\left(t, x_{t}\right) \leq-w_{i}(\|x(t)\|)<-\gamma_{i}(\eta), \quad \forall t \geq t_{0}
$$


This implies

$$
\begin{aligned}
V_{i}\left(t, x_{t}\right) & \leq V_{i}\left(t_{0}, \varphi\right)-\left(t-t_{0}\right) \gamma_{i}(\eta) \\
& \leq v_{i}\left(\delta_{1}\right)-\left(t-t_{0}\right) \gamma_{i}(\eta) .
\end{aligned}
$$

Let $T_{*}>\left[v_{i}\left(\delta_{1}\right)\right] / \gamma_{i}$. Then for every $t>t_{0}+T_{*}$, we have $V_{i}\left(t, x_{t}\right) \leq 0$. However, we assume that there is a time instant $t_{1}>t_{0}+T_{*}$ such that $\left\|x\left(t_{1}\right)\right\|>\eta$. This implies that

$$
V_{i}\left(t_{1}, x_{t_{1}}\right) \geq u_{i}\left(\left\|x\left(t_{1}\right)\right\|\right) \geq u_{i}(\eta)>0
$$

This is a contradiction. Therefore time instant $t_{1}$ cannot exits and this implies

$$
\|x(t)\| \leq \eta, \quad \forall t>t_{0}+\frac{v_{i}\left(\delta_{1}\right)}{\gamma_{i}(\eta)} .
$$

Similarly, assuming there is a time instant $t_{1}>t_{0}+T_{*}$ such that $\left\|\dot{x}\left(t_{1}\right)\right\| \geq \eta$

$$
V_{i}\left(t_{1}, x_{t_{1}}\right) \geq u_{d_{i}}\left(\left\|\dot{x}\left(t_{1}\right)\right\|\right) \geq u_{d_{i}}(\eta)>0
$$

which is also a contradiction. Hence,

$$
\|x(t)\|<\eta, \quad\|\dot{x}(t)\|<\eta, \quad \forall t>t_{0}+T_{*}
$$

If we wait for a period of maximum time-delay such that $t>t_{0}+T_{*}+\tau_{\max }$, the inequality $|x(t)|_{\left[t-\tau_{\max }, t\right]} \leq \eta$ holds, which concludes the proof.

Proposition 2. Consider the system (1) with each $\Sigma_{i}$ satisfying Lemma 2, if there exist matrices $W_{i}^{T}=W_{i} \succeq 0$ such that following LMIs hold,

$$
\bar{\phi}_{i}:=\left[\begin{array}{ccc}
\phi_{11 i}+W_{i} & \phi_{12 i} & \tau_{i} A_{i}^{T} Z_{i} \\
* & \phi_{22 i} & \tau_{i} \bar{A}_{i}^{T} Z_{i} \\
* & * & -\tau_{i} Z_{i}
\end{array}\right] \prec 0,
$$

then $\dot{V}_{i}\left(t, x_{t}\right) \leq-x^{T}(t) W_{i} x(t)$.

Proof. Consider the inequality (8). Since $\psi_{i} \succeq 0$, we know that $\dot{V}_{i}\left(t, x_{t}\right) \leq \xi_{1}^{T}(t) \Xi_{i} \xi_{1}(t)$. Bounding this inequality,

$$
\xi_{1}^{T}(t) \Xi_{i} \xi_{1}(t) \leq-x^{T}(t) W_{i} x(t)
$$

yields $\xi_{1}^{T}(t) D_{i} \xi_{1}(t) \leq 0$ where

$$
D_{i}:=\left[\begin{array}{cc}
\phi_{11 i}+W_{i}+\tau_{i} A_{i}^{T} Z_{i} A_{i} & \phi_{12 i}+\tau_{i} A_{i}^{T} Z_{i} \bar{A}_{i} \\
* & \phi_{22 i}+\tau_{i} \bar{A}_{i}^{T} Z_{i} \bar{A}_{i}
\end{array}\right] .
$$

Since $\bar{\phi}_{i}$ is the Schur Complement of $D_{i}$, if (9) holds, then $\dot{V}_{i}\left(t, x_{t}\right) \leq-x^{T}(t) W_{i} x(t)$.

Then we can define the upper bound for the derivative of the Lyapunov function in lemma 1 as $w_{i}\left(s^{2}\right):=$ $\sigma_{\min }\left[W_{i}\right] s^{2}$.

Theorem 1. Consider the switched delay system described in (1). Assume all of the candidate subsystems satisfy lemma 2. Then, the switched delay system is asymptotically stable for all switching signals satisfying dwell time requirement $\tau_{D}$

$$
\tau_{D}=\frac{1}{\alpha^{2}} \max _{i \in \mathcal{P}} \frac{v_{i}}{w_{i}}+\max _{i \in \mathcal{P}} \tau_{i}, \quad \text { for any } \alpha \in(0,1)
$$

where

$$
\begin{aligned}
v_{i} & =\sigma_{\max }\left[P_{i}\right]+\tau_{i} \sigma_{\max }\left[Q_{i}\right]+\frac{1}{2} \tau_{i}^{2} \sigma_{\max }\left[Z_{i}\right], \\
w_{i} & =\sigma_{\min }\left[W_{i}\right]
\end{aligned}
$$

Proof. Let's choose $\eta=\alpha \delta_{k}$ where $\delta_{k}$ denotes norm of the state at the $k^{t h}$ switching instant such that $\delta_{k}=$ $|x|_{\left[t_{k}-\tau_{\max }, t_{k}\right]}$. Introducing the dwell time as

$$
\tau_{D}=\max _{i \in \mathcal{P}} \tau_{i}+\max _{i \in \mathcal{P}} T_{i}(\eta)
$$

leads us to an inequality from proposition 1 as following,

$$
|x|_{\left[t_{k}-\tau_{\max }, t_{k}\right]} \leq \alpha|x|_{\left[t_{k-1}-\tau_{\max }, t_{k-1}\right]}, \quad \forall t_{k}>t_{k-1}+\tau_{D}
$$

where

$$
T_{i}(\eta)=T_{i}\left(\alpha \delta_{k}\right)=\frac{v_{i}}{\alpha^{2} w_{i}} .
$$

From (4), we know that

$$
\|x(t)\| \leq \sqrt{\frac{v_{i}}{u_{i}}}|x|_{\left[t_{k}-\tau_{\max }, t_{k}\right]}
$$

for any $i \in \mathcal{P}$. Let's define

$$
\beta=\max _{i \in \mathcal{P}} \sqrt{\frac{v_{i}}{u_{i}}} .
$$

Then,

$$
\begin{aligned}
\|x(t)\| \leq & \beta|x|_{\left[t_{k}-\tau_{\max }, t_{k}\right]} \\
\leq & \beta \alpha|x|_{\left[t_{k-1}-\tau_{\max }, t_{k-1}\right]} \\
& \quad \vdots \\
\leq & \beta \alpha^{k}|x|_{\left[t_{0}-\tau_{\max }, t_{0}\right]} \quad \forall \alpha \in(0,1) \\
\leq & \beta \alpha|x|_{\left[t_{0}-\tau_{\max }, t_{0}\right]}, \quad \forall \alpha \in \text {. }
\end{aligned}
$$

which is satisfying the stability condition described in definition 1 .

Remark 1. The parameter $\alpha$ can be regarded as a measure of the decay rate. This parameter quantifies a trade-off between the dwell time and the decay rate, i.e.; the larger $\alpha$, the smaller dwell time but the slower decay rate.

\section{Minimum Dwell Time via Quasi-ConveX OPTIMIZATION}

In order to minimize dwell time given by (10), the cost function $f\left(v_{i}, w_{i}\right):=\max _{i \in \mathcal{P}} v_{i} / w_{i}$ should be minimized. This is a quasi-convex function since it is the composition of a convex function with a nondecreasing function [2]. It is known that an optimization problem with a quasiconvex cost function and convex constraints can be solved by iterative methods such as bisection algorithm [1]. We define a parameter $t$ to denote an upper bound for the cost function such that $f\left(v_{i}, w_{i}\right) \leq t$.

Let's define

$$
X_{i}:=\left[\begin{array}{cc}
X_{11 i} & X_{12 i} \\
* & X_{21 i}
\end{array}\right] .
$$


For the free parameters $P_{i}, Q_{i}, Z_{i}, W_{i}, X_{11 i}, X_{12 i}, X_{21 i}$, $N_{1 i}, N_{2 i} p_{i}, q_{i}, z_{i}, w_{i}, t$, minimum dwell time can be computed via following optimization problem:

$$
\begin{array}{ll}
\min & t \\
\text { s.t. } & \operatorname{diag}\left[P_{i}, Q_{i}, Z_{i}, W_{i}, X_{i}\right] \succ 0, \\
& \operatorname{diag}\left[P_{i}, Q_{i}, Z_{i},-W_{i}\right] \prec \operatorname{diag}\left[p_{i} I, q_{i} I, z_{i} I,-w_{i} I\right] \\
& \psi_{i} \succeq 0, \quad \bar{\phi}_{i} \prec 0 \\
& p_{i}+\tau_{i} q_{i}+\frac{1}{2} \tau_{i}^{2} z_{i}-t w_{i}<0, \quad \forall i \in \mathcal{P}
\end{array}
$$

where $\psi_{i}$ and $\bar{\phi}_{i}$ are defined in (7) and (9), respectively. Then, dwell time is $\tau_{D}=t+\tau_{\max }$. However, optimization problem in (11) involves a bilinear matrix inequality when $t$ is considered as a free parameter.

Searching for minimum $t$ with bisection algorithm generates a sequence of linear semi-definite programming (SDP) feasibility problems which can easily be solved by SeDuMi [17].

\section{NUMERICAL EXAMPLES}

In this section, the examples are taken from [3] and [20] for comparison purposes.

Example 1. Let $\Sigma_{1}$ be

$$
\begin{aligned}
A_{1}=\left[\begin{array}{cc}
-2 & 0 \\
0 & -0.9
\end{array}\right], \quad \bar{A}_{1}=\left[\begin{array}{cc}
-1 & 0 \\
-0.5 & -1
\end{array}\right], \\
\tau_{1}=0.3 s, \quad d_{1}=0 .
\end{aligned}
$$

and let $\Sigma_{2}$ be

$$
\begin{aligned}
& A_{2}=\left[\begin{array}{cc}
-1 & 0.5 \\
0 & -1
\end{array}\right], \quad \bar{A}_{2}=\left[\begin{array}{cc}
-1 & 0 \\
0.1 & -1
\end{array}\right], \\
& \tau_{2}=0.6 s, \quad d_{2}=0 .
\end{aligned}
$$

Corresponding minimum dwell times for different $\tau_{i}$ and $d_{i}$ values are illustrated in table II.

Example 2. Let $\Sigma_{1}$ be

$$
\begin{aligned}
A_{1}=\left[\begin{array}{cc}
-1.799 & -0.814 \\
0.2 & -0.714
\end{array}\right], & \bar{A}_{1}=\left[\begin{array}{cc}
-1 & 0 \\
-0.45 & -1
\end{array}\right], \\
& \tau_{1}=0.155 s, \quad d_{1}=0 .
\end{aligned}
$$

and let $\Sigma_{2}$ be

$$
\begin{aligned}
A_{2}=\left[\begin{array}{cc}
-1.853 & -0.093 \\
-0.853 & -1.1593
\end{array}\right], \quad \bar{A}_{2}=\left[\begin{array}{cc}
-1 & 0 \\
0.05 & -1
\end{array}\right], \\
\tau_{2}=0.2 s, \quad d_{2}=0 .
\end{aligned}
$$

Comparison of present paper with previous works for examples 1 and 2 can be seen in table I. Corresponding minimum dwell times for different $\tau_{i}$ and $d_{i}$ values are illustrated in table III.
TABLE I

DWELL TIME FOR $\alpha=0.99$

\begin{tabular}{rrrc}
\hline Ex. & Paper [20] & Paper [3] & Present Paper \\
\hline 1 & $6.51 \mathrm{~s}$ & $3.4 \mathrm{~s}$ & $1.11 \mathrm{~s}$ \\
2 & - & $0.72 \mathrm{~s}$ & $0.58 \mathrm{~s}$ \\
\hline
\end{tabular}

TABLE II

DWELl TIME FOR DIFFERENT $\tau_{i}$ AND $d_{i}$ VALUES OF EXAMPLE 1

\begin{tabular}{rrrrr}
\hline$\tau_{1}$ & $\tau_{2}$ & $d_{1}$ & $d_{2}$ & $\tau_{D}$ \\
\hline $0.15 \mathrm{~s}$ & $0.3 \mathrm{~s}$ & $0 \mathrm{~s}$ & $0 \mathrm{~s}$ & $0.69 \mathrm{~s}$ \\
$0.15 \mathrm{~s}$ & $0.3 \mathrm{~s}$ & $0.15 \mathrm{~s}$ & $0.3 \mathrm{~s}$ & $0.69 \mathrm{~s}$ \\
$0.3 \mathrm{~s}$ & $0.6 \mathrm{~s}$ & $0 \mathrm{~s}$ & $0 \mathrm{~s}$ & $1.11 \mathrm{~s}$ \\
$0.3 \mathrm{~s}$ & $0.6 \mathrm{~s}$ & $0.3 \mathrm{~s}$ & $0.3 \mathrm{~s}$ & $1.11 \mathrm{~s}$ \\
$0.3 \mathrm{~s}$ & $0.6 \mathrm{~s}$ & $0.6 \mathrm{~s}$ & $0.6 \mathrm{~s}$ & $1.11 \mathrm{~s}$ \\
$0.6 \mathrm{~s}$ & $1.2 \mathrm{~s}$ & $0 \mathrm{~s}$ & $0 \mathrm{~s}$ & $2.54 \mathrm{~s}$ \\
$0.6 \mathrm{~s}$ & $1.2 \mathrm{~s}$ & $0.3 \mathrm{~s}$ & $0.3 \mathrm{~s}$ & $2.76 \mathrm{~s}$ \\
$0.6 \mathrm{~s}$ & $1.2 \mathrm{~s}$ & $0.6 \mathrm{~s}$ & $0.6 \mathrm{~s}$ & $3.51 \mathrm{~s}$ \\
\hline
\end{tabular}

TABLE III

DWELL TIME FOR DIFFERENT $\tau_{i}$ AND $d_{i}$ VALUES OF EXAMPLE 2

\begin{tabular}{rrrrr}
\hline$\tau_{1}$ & $\tau_{2}$ & $d_{1}$ & $d_{2}$ & $\tau_{D}$ \\
\hline $0.08 \mathrm{~s}$ & $0.1 \mathrm{~s}$ & $0 \mathrm{~s}$ & $0 \mathrm{~s}$ & $0.46 \mathrm{~s}$ \\
$0.155 \mathrm{~s}$ & $0.2 \mathrm{~s}$ & $0 \mathrm{~s}$ & $0 \mathrm{~s}$ & $0.58 \mathrm{~s}$ \\
$0.155 \mathrm{~s}$ & $0.2 \mathrm{~s}$ & $0.15 \mathrm{~s}$ & $0.15 \mathrm{~s}$ & $0.58 \mathrm{~s}$ \\
$0.3 \mathrm{~s}$ & $0.4 \mathrm{~s}$ & $0 \mathrm{~s}$ & $0 \mathrm{~s}$ & $0.84 \mathrm{~s}$ \\
$0.3 \mathrm{~s}$ & $0.4 \mathrm{~s}$ & $0.2 \mathrm{~s}$ & $0.2 \mathrm{~s}$ & $0.84 \mathrm{~s}$ \\
$0.6 \mathrm{~s}$ & $0.8 \mathrm{~s}$ & $0 \mathrm{~s}$ & $0 \mathrm{~s}$ & $1.38 \mathrm{~s}$ \\
$0.9 \mathrm{~s}$ & $1.2 \mathrm{~s}$ & $0 \mathrm{~s}$ & $0 \mathrm{~s}$ & $1.38 \mathrm{~s}$ \\
$0.9 \mathrm{~s}$ & $1.2 \mathrm{~s}$ & $0.3 \mathrm{~s}$ & $0.3 \mathrm{~s}$ & $2.39 \mathrm{~s}$ \\
$0.9 \mathrm{~s}$ & $1.2 \mathrm{~s}$ & $0.6 \mathrm{~s}$ & $0.6 \mathrm{~s}$ & $3.15 \mathrm{~s}$ \\
$0.9 \mathrm{~s}$ & $1.2 \mathrm{~s}$ & $0.9 \mathrm{~s}$ & $0.9 \mathrm{~s}$ & $176.70 \mathrm{~s}$ \\
\hline
\end{tabular}

\section{CONCLUSIONS}

We performed the calculation of minimum dwell time to ensure stability of switched delay systems. Minimization of dwell time is formulated as a quasi-convex optimization problem. Stability conditions are derived by using free weighting matrices method to find appropriate LyapunovKrasovskii functionals. By the numerical examples, it is shown that the results obtained in [3] and [20] can be improved using the method proposed in the present paper.

\section{REFERENCES}

[1] S. Boyd and L. Vandenberghe. Convex Optimization. Cambridge University Press, Cambridge, 2004.

[2] F. Bullo and D. Liberzon. Quantized control via locational optimization. IEEE Transactions on Automatic Control, 51(1):2-13, 2006.

[3] S. Y. Çalışkan, H. Özbay, and S.-I. Niculescu. Dwell-time computation for stability of switched systems with time delays. IET Control Theory \& Applications, 7(10):1422-1428, 2013.

[4] L. Fainshil, M. Margaliot, and P. Chigansky. On the stability of positive linear switched systems under arbitrary switching laws. IEEE Transactions on Automatic Control, 54(4):897-899, April 2009.

[5] K. Gu, V. Kharitonov, and J. Chen. Stability of Time-Delay Systems. Birkhauser, Boston, 2003.

[6] Y. He, Q.-G. Wang, L. Xie, and C. Lin. Further improvement of free-weighting matrices technique for systems with time-varying delay. IEEE Transactions on Automatic Control, 52(2):293-299, Feb 2007.

[7] J. P. Hespanha. Uniform stability of switched linear systems: extensions of lasalle's invariance principle. IEEE Transactions on Automatic Control, 49(4):470-482, 2004. 
[8] J.P. Hespanha and A.S. Morse. Stability of switched systems with average dwell-time. In Proceedings of the 38th IEEE Conference on Decision and Control, volume 3, pages 2655-2660 vol.3, 1999.

[9] M. Hou, F. Fu, and G. Duan. Global stabilization of switched stochastic nonlinear systems in strict-feedback form under arbitrary switchings. Automatica, 49(8):2571 - 2575, 2013.

[10] Z. Li, H. Gao, R. Agarwal, and O. Kaynak. $H_{\infty}$ control of switched delayed systems with average dwell time. International Journal of Control, 86(12):2146-2158, 2013.

[11] D. Liberzon and A. S. Morse. Basic problems in stability and design of switched systems. IEEE Control Systems, 19(5):59-70, Oct 1999.

[12] H. Lin and P. J. Antsaklis. Stability and stabilizability of switched linear systems: A survey of recent results. IEEE Transactions on Automatic Control, 54(2):308-322, Feb 2009.

[13] M. S. Mahmoud. Switched Time-Delay Systems. Springer-Verlag, Boston, 2010.

[14] S. Mitra and D. Liberzon. Stability of hybrid automata with average dwell time: an invariant approach. In 43rd IEEE Conference on Decision and Control, volume 2, pages 1394-1399 Vol.2, Dec 2004.

[15] A.S. Morse. Supervisory control of families of linear set-point controllers part i. exact matching. IEEE Transactions on Automatic Control, 41(10):1413-1431, Oct 1996.

[16] R. Shorten, K.S. Narendra, and O. Mason. A result on common quadratic lyapunov functions. IEEE Transactions on Automatic Control, 48(1):110-113, Jan 2003.

[17] J. F. Sturm. Using sedumi 1.02, a matlab toolbox for optimization over symmetric cones. Optimization Methods and Software, 11(1-4):625653, 1999.

[18] X.-M. Sun, J. Zhao, and D. J. Hill. Stability and $L_{2}$-gain analysis for switched delay systems: A delay-dependent method. Automatica, 42(10): 1769 - 1774, 2006.

[19] M. Wu, Y. He, and J.-H. She. Stability Analysis and Robust Control of Time-Delay Systems. Science Press Beijing and Springer-Verlag, London, 2010

[20] P. Yan and H. Özbay. Stability analysis of switched time delay systems. SIAM Journal on Control and Optimization, 47(2):936-949, 2008.

[21] J. Zhang, Z. Han, F. Zhu, and J. Huang. Stability and stabilization of positive switched systems with mode-dependent average dwell time. Nonlinear Analysis: Hybrid Systems, 9(0):42 - 55, 2013. 